\title{
A Comparative Study of Enamel Surface Roughness After Bleaching With Diode Laser and Nd: YAG Laser
}

\author{
Mansoreh Mirzaie', Esmaiel Yassini ${ }^{1}$, Saber Ganji ${ }^{2}$, Zohreh Moradi ${ }^{*}$, Nasim Chiniforush ${ }^{3}$ \\ ${ }^{1}$ Department of Operative Dentistry, Tehran University of Medical Sciences, Tehran, Iran \\ ${ }^{2}$ Private Practice, Tehran, Iran \\ ${ }^{3}$ Laser Research Center of Dentistry (LRCD), Dental Research Institute, Tehran University of Medical Sciences, Tehran, Iran
}

\author{
*Correspondence to \\ Zohreh Moradi, DDS; Department \\ of Operative Dentistry, Tehran \\ University of Medical Sciences, \\ Tehran, Iran. \\ Tel: $+98-2188015017$; \\ Fax: +98-2188015017 \\ Email: z-moradi@sina.tums.ac.ir
}

Published online 18 July 2016

\begin{abstract}
Introduction: Bleaching process can affect surface roughness of enamel, which is a vital factor in esthetic and resistance of tooth. The aim of this study was to compare surface roughness of enamel in teeth bleached using Diode and Neodymium-Doped Yttrium Aluminium Garnet (Nd: YAG) lasers with those bleached using conventional method.

Methods: In this study, 75 anterior human teeth from upper and lower jaws (These teeth extracted because of periodontal disease) were randomly divided into 5 groups. Group 1: Laser white gel (Biolase, USA) with $45 \%$ hydrogen peroxide concentration and GaAlAs Diode laser (CHEESE ${ }^{\mathrm{TM}}$, GIGAA, China), group 2: Heydent gel (JW, Germany) with 30\% Hydrogen peroxide concentration and Diode laser, group 3: Laser white gel and $\mathrm{Nd}$ :YAG laser (FIDELIS ${ }^{\text {TM }}$, Fotona, Slovenia), group 4: Heydent gel and Nd:YAG laser and group 5: The Iranian gel Kimia (Iran) with 35\% hydrogen peroxide concentration were used. Surface roughness of the samples was measured using the Surface Roughness Tester system (TR 200 Time Group, Germany) before and after bleaching. In each group, one sample was randomly selected for SEM analysis.

Results: The results showed that the mean surface roughness of the teeth before and after bleaching had a significant difference in all the study groups. It was indicated that after bleaching, the mean surface roughness had increased in all the study groups. The highest surface roughness was seen in the conventional bleaching group and the lowest surface roughness was reported in group 3 (laser white gel + diode laser), in which the average surface roughness increased by only $0.1 \mu \mathrm{m}$.

Conclusion: It was concluded that using the Laser white gel and the diode laser for bleaching resulted in the least surface roughness compared to conventional method.

Keywords: bleaching; micro roughness; hydrogen peroxide; laser.
\end{abstract}

\section{Introduction}

Today, there is a worldwide trend towards esthetic treatments for tooth discoloration. Extrinsic or intrinsic stains can cause tooth discoloration. While polishing with rubber cap is used to easily remove extrinsic stains, bleaching, veneers, laminates and ceramic restorations are used to treat intrinsic discoloration. ${ }^{1}$

Bleaching is the most conservative esthetic treatment for tooth discoloration which has some advantages compared to other treatments like cost efficiency and no need for tooth preparation. ${ }^{2}$

In-office bleaching is carried out using hydrogen peroxide at concentrations ranging $30 \%$ to $40 \%$, or carbamide peroxide $35 \%$ to $37 \%$. Light sources are being used to increase the free radicals of bleaching agents in the process. Today, due to the high demand of patients for quick and effective results, lasers such as diodes, Neodymium-Doped Yttrium Aluminium Garnet (Nd: YAG) and Erbium-Doped Yttrium Aluminium Garnet (Er: YAG) laser have been introduced as light sources.

Bleaching treatment may cause morphological changes and demineralization in mineralized structures. ${ }^{3,4}$ This alteration in tooth structure can lead to change in enamel micro-hardness and micro-roughness, ${ }^{5}$ which can be assessed by micro-hardness and micro-roughness tests. These changes in the enamel are still a controversial and conflicting subject in the literature. ${ }^{6}$

Bitter in its study concluded that bleaching can cause obvious changes in the enamel morphology, such as decreased thickness of exposed prismless enamel, demineralization of enamel prisms and increased surface porosity. ${ }^{7}$ Although Xu et al did not detect any morphological and surface chemical composition changes after bleaching with neutral and alkaline bleaching solutions. ${ }^{8}$

If overbleaching happens, some side effects such as brittleness and increased surface porosity can be produced. Also, increasing the concentration of $\mathrm{H}_{2} \mathrm{O}_{2}$ enhances the possibility of external root resorption. ${ }^{9}$ 
Hence the aim of this study was to evaluate and compare micro-roughness of the enamel of teeth bleached using the diode and Nd: YAG lasers with those bleached using regular method.

\section{Methods}

In this study, 75 maxillary and mandibular anterior teeth with no caries or cracks were collected. These teeth extracted because of periodontal disease. The specimens were stored in normal saline solution at $37^{\circ} \mathrm{C}$ after extraction. The teeth were mounted in acrylic resin so that buccal surfaces were exposed, then their pre-treatment buccal surface micro-roughness was evaluated with a profilometer (Surface Roughness Tester, TR 200 Time group Germany) and they were divided into 5 random groups as below:

Group 1: Bleaching with diode laser and Heydent gel (30\% Hydrogen peroxide concentration),

Group 2: Bleaching with Nd: YAG laser and Heydent gel, Group 3: Bleaching with diode laser and laser white gel (45\% hydrogen peroxide concentration),

Group 4: Bleaching with Nd: YAG laser and laser white gel, and

Group 5: Bleaching with Kimia gel (35\% hydrogen peroxide concentration).

In group 1, the Heydent gel (production of Farafan Diagnostics Co, under license of Heydent, Germany Tehran - Iran) with a $1 \mathrm{~mm}$ thickness was placed on the buccal surface of the tooth, then diode laser (Wuhan Gigaa Optronics Technology Co, LTD, China) was applied on the gel for 30 seconds $(810 \mathrm{~nm}, 1.5 \mathrm{~W}, 400 \mu$ fiber, continuous, $6 \mathrm{~mm}$ distance from the surface). After a 60 seconds interval, the process was repeated and after that, the Heydent gel was left on the buccal surface for 3 minutes and then washed with distilled water.

In group 2, the Heydent gel with a $1 \mathrm{~mm}$ thickness was placed on the buccal surface of the tooth, then Nd: YAG laser (FIDELIS, Fotona, Slovenia) was applied on the gel for 30 seconds $(2.5 \mathrm{~W}, 25 \mathrm{~Hz}, 320 \mu$ fiber, $6 \mathrm{~mm}$ distance from the surface). After a 60 seconds interval, the process was repeated and after that, the Heydent gel was left on the buccal surface for 3 minutes and then washed with distilled water.

In group 3, the laser white gel ( Laser White 20, Biolase, USA) with a $1 \mathrm{~mm}$ thickness was placed on the buccal surface of the tooth, then diode laser was applied on the gel for 30 seconds $(810 \mathrm{~nm}, 1.5 \mathrm{~W}, 400 \mu$ fiber, continuous, $6 \mathrm{~mm}$ distance from the surface). After a 60 seconds interval, the process was repeated and after that, the laser white gel was left on the buccal surface for 3 minutes and then washed with distilled water.

In group 4, the laser white gel with a $1 \mathrm{~mm}$ thickness was placed on the buccal surface of the tooth, then Nd: YAG laser was applied on the gel for 30 seconds $(2.5 \mathrm{~W}, 25 \mathrm{~Hz}$, $320 \mu$ fiber, $6 \mathrm{~mm}$ distance from the surface). After a 60 seconds interval, the process was repeated and after that, the white laser gel was left on the buccal surface for $3 \mathrm{~min}$ utes and then washed with distilled water.
In group 5, only the Kimia gel (Kimia, Iran) was placed on the buccal surface for 15 minutes and then washed with distilled water.

The procedure of bleaching took 7 minutes and $30 \mathrm{sec}$ onds in groups 1, 2, 3 and 4. All the specimens were dried and then the enamel micro-roughness of the teeth was evaluated using a profilometer.

One specimen from each group was randomly selected for the electron microscope study (jsm829, Jeol, Tokyo, Japan, 2000×).

Data were statistically analyzed using SPSS 15.0 package. One-way analysis of variance (ANOVA) and Tukey post hoc tests were used to test the significance for the effects of bleaching agent on surface roughness at $P \leq 0.05$

\section{Results}

The micro-roughness mean values of the 5 study groups before bleaching are shown in Table 1. The ANOVA test did not indicate any significant difference between micro-roughness mean values in all 5 groups before bleaching $\left(\mathrm{F}_{4,70}=0.102, P=0.98\right)$. The micro-roughness mean value in all 5 groups was $1.54 \pm 0.48 \mu \mathrm{m}$.

The micro-roughness mean values of the 5 study groups after bleaching are shown in Table 2. According to the ANOVA test results, the micro-roughness mean value of each group, before and after bleaching showed a significant difference. There was an increase in micro-roughness value after bleaching in all 5 groups compared to the micro-roughness of specimens before bleaching which means bleaching can increase the micro-roughness of enamel regardless of the procedure. The highest increase in micro-roughness belonged to group 5 (bleached with conventional method) and the lowest increase belonged to group 3 (bleached with diode laser and white laser gel). According to the Tukey post hoc test results, the lowest micro-roughness value belonged to group 3 (bleached with diode laser and white laser gel). This group showed a significant difference with all the other study groups ex-

Table 1. The Micro-Hardness Mean Values of the 5 Study Groups Before Bleaching

\begin{tabular}{ll}
\hline $\begin{array}{l}\text { Mean } \\
(\boldsymbol{\mu m})\end{array}$ & Group \\
\hline 1.57 & Group 1 (Bleaching with diode laser and Heydent gel) \\
1.56 & Group 2 (Bleaching with Nd: YAG laser and Heydent gel) \\
1.51 & Group 3 (Bleaching with diode laser and Biolase gel) \\
1.54 & Group 4 (Bleaching with Nd: YAG laser and Biolase gel) \\
1.52 & Group 5 or control (Bleaching with Kimia gel) \\
\hline
\end{tabular}

Table 1. The Micro-Hardness Mean Values of the 5 Study Groups After Bleaching

\begin{tabular}{ll}
\hline $\begin{array}{l}\text { Mean } \\
(\mu \mathrm{m})\end{array}$ & Group \\
\hline 1.98 & Group 1 (Bleaching with diode laser and Heydent gel) \\
2.18 & Group 2 (Bleaching with Nd: YAG laser and Heydent gel) \\
1.61 & Group 3 (Bleaching with diode laser and Biolase gel) \\
1.76 & Group 4 (Bleaching with Nd: YAG laser and Biolase gel) \\
2.33 & Group 5 or control (Bleaching with Kimia gel) \\
\hline
\end{tabular}


cept for the group 4 (bleached with $\mathrm{Nd}$ : YAG laser and white laser gel).The results also indicated that there was a significant difference between group 5 (conventional bleaching) with all the other groups except for the group 2 (bleached with Nd: YAG laser and Heydent gel). There was not a significant difference between group 1 (bleaching with diode laser and Heydent gel) and group 2 (bleached with Nd: YAG laser and Heydent gel) $(P=0.73)$, although these 2 groups had significantly higher micro-roughness compared to the groups 3 and 4 (bleached with diode laser and White laser gel, bleached with Nd: YAG laser and White laser gel) $(P=0.01)$.

The SEM results were in accordance with micro roughness results, bleaching with laser white gel and diode laser increased the lowest surface roughness.

\section{Discussion}

Today, bleaching is a renowned esthetic process for discoloration treatment. It is a procedure to remove stains from dental structure. ${ }^{3}$ Hydrogen peroxide has long been used as a gel in bleaching process. Bleaching treatment can change the enamel micro-hardness and micro-roughness. ${ }^{5}$ In this study, we evaluated 2 bleaching gel (Heydent and white laser) with 2 light sources (Nd:YAG and diode) and compared them with conventional bleaching (Kimia gel). The result of this study indicated that using diode laser would lead to less micro-roughness compared to the conventional method.

Different studies have evaluated various light sources in the bleaching process and some of them indicated that using laser as a light source would lead to better results. ${ }^{10}$ In this study, it was concluded that using laser in bleaching would take less contact time between gel and tooth surface, and therefore it would produce more favorable results regarding enamel micro-roughness. It was concluded that white gel produces more favorable results compared to Heydent gel and also diode laser as a light source leads to less enamel micro-roughness compared to Nd: YAG laser. The highest micro-roughness in conventional method was due to the high $\mathrm{pH}$ level of Kimia gel used in the process.

The results of this study were in accordance with the studies of Pinto et al, ${ }^{11}$ Dominguez et al, ${ }^{12}$ De Vasconcelos et al, ${ }^{13}$ and Sasaki et al, ${ }^{14}$ which reported that in-office bleaching increases surface roughness of enamel. The results of the present study were in contrast with the results of McGuckin et al, ${ }^{15}$ Mondelli et $\mathrm{al}^{16}$ and Borges et al ${ }^{17}$ which indicated that in conventional bleaching procedure, the enamel micro-roughness could not increase. The discrepancy in these results may have been caused by different light sources, different tooth type selection or other material in the study and also storage of specimens in saliva. In this study, the specimens were stored in distilled water which does not have the remineralizing effect of saliva due to fluoride. Also in this in vitro study anterior teeth that were extracted because of periodontal disease, were studied and hence the results could be somehow different compared to clinical conditions.
Bleaching can cause structural changes in the enamel too, besides the change in surface micro-roughness. The alteration in the rate of absorbed water and the disintegration in the enamel surface can lead to the stain accumulation and discoloration. ${ }^{17-21}$

When the surface roughness of enamel is increased, some adversities are expected as result, such as decrease in enamel resistance, increase in enamel brittleness, permeability and stain and plaque accumulation..$^{22-24}$

Some methods have recommended to increase gel concentration or working time in order to get more effective results in bleaching. In this study, we were searching for a bleaching method that could have the least adverse effects on teeth, while helping us use a less concentrated gel in a less working time to achieve safer and more favorable result.

Using diode laser as a light source in bleaching has some benefits such as less working time due to its in-depth activity, more efficiency and safety due to its reaction with specific chromophores in the bleaching gel. ${ }^{25}$

Regarding the limitations of this study and other previous in vitro ones, it is necessary that further researches take place to find a bleaching method with minimal side effects, simulating the clinical conditions.

\section{Conclusion}

Considering the limitations of this in vitro study it was concluded that white laser gel produces more favorable results compared to Heydent gel and also diode laser as a light source leads to less enamel micro-roughness compared to Nd: YAG laser. Therefore bleaching with white laser gel and diode laser would lead to the most favorable results while conventional bleaching increases enamel surface roughness the most.

It is recommended that further researches study clinical situations and in-vivo conditions such as storing specimens in saliva instead of distilled water. Also it is suggested to evaluate carbamide peroxide besides hydrogen peroxide in the future studies.

\section{Ethical Considerations}

In this in vitro study, there was no ethical conflict.

\section{Conflict of Interests}

The authors whose names are listed in this article certify that they have no affiliations with or involvement in any organization or entity with any financial interest or non-financial interest in the subject matter or materials discussed in this manuscript.

\section{References}

1. Leonard RH, Haywood VB, Eagle JC, et al. Nightguard vital bleaching of tetracycline-stained teeth: 54 months post treatment. J Esthet Dent. 1999;11:265-277. doi:10.1111/j.1708-8240.1999.tb00408.x.

2. Ahamed S, Thayyil S, Sathyanarayana SS, Kulandaivelu A, Satheesh Kumar KS, Deepa VK. The effect of power bleaching on enamel microhardness activated by three different light sources: an in vitro study. J Dent Lasers. 
2013;7:48-53. doi:10.4103/0976-2868.124262.

3. Goldstein RE, Garber DA. Complete Dental Bleaching. Chicago: Quintessence Books; 1995. 3.

4. Fasanaro TS. Bleaching teeth: History, chemicals, and methods used for common tooth discolorations. J Esthet Dent. 1992;4:71-78. doi:10.1111/j.1708-8240.1992. tb00666.x.

5. Marcondes M, Paranhos MP, Spohr AM, et al. The influence of the Nd:YAG laser bleaching on physical and mechanical properties of the dental enamel. J Biomed Mater Res B Appl Biomater. 2009;90(1):388-395. doi:10.1002/jbm.b.31297.

6. Cadenaro M, Breschi L, Nucci C, et al . Effect of two inoffice whitening agents on the enamel surface in vivo: a morphological and non-contact profilometric study. Oper Dent. 2008; 33(2):127-134. doi:10.2341/07-89.

7. Bitter NC. A scanning electron microscope study of the long-term effect of bleaching agents on the enamel surface in vivo. Gen Dent. 1998;46(1):84-8.

8. Xu B, Li Q, Wang Y. Effects of $\mathrm{pH}$ values of hydrogen peroxide bleaching agents on enamel surface properties. Oper Dent. 2011;36(5):554-62. doi:10.2341/11-045-1.

9. Berger SB, Cavalli V, Ambrosano GM, Giannini M. Changes in surface morphology and mineralization level of human enamel following in-office bleaching with $35 \%$ hydrogen peroxide and light irradiation. Gen Dent. 2010;58(2):e74-e79.

10. Joiner A. The bleaching of teeth: a review of the literature. J Dent. 2006;34(7):412-419. doi:10.1016/j.jdent.2006.02.002.

11. Pinto CF, de Oliveira R, Cavalli V, Giannini M. Peroxide bleaching agent effects on enamel surface microhardness, roughness and morphology. Braz Oral Res. 2004;18(4):306-311. doi:10.1590/s180683242004000400006 .

12. Dominguez JA, Bittencourt B, Michel M, Sabino N, Gomes JC, Gomes OM . Ultrastructural evaluation of enamel after dental bleaching associated with fluoride. Microsc Res Tech. 2012;75(8):1093-1098. doi:10.1002/jemt.22035.

13. De Vasconcelos AA, Cunha AG, Borges BC, Vitoriano Jde O, Alves- Junior C, Machado CT . Enamel properties after tooth bleaching with hydrogen/carbamide peroxides in association with a CPP-ACP paste. Acta Odontol Scand. 2012; 70(4):337-343.

14. Sasaki RT, Arcanjo AJ, Florio FM, Basting RT.
Micromorphology and microhardness of enamel after treatment with home-use bleaching agents containing $10 \%$ carbamide peroxide and $7.5 \%$ hydrogen peroxide. J Appl Oral Sci. 2009;17(6):611-616. doi:10.1590/s167877572009000600014.

15. McGuckin RS, Babin JF, Meyer BJ. Alterations in human enamel surface morphology following vital bleaching. J Prosth Dent. 1992;68(5):754-760. doi:10.1016/00223913(92)90197-i.

16. Mondelli RF, Azevedo JF, Francisconi PA, Ishikiriama SK, Mondelli J. Wear and surface roughness of bovine enamel submitted to bleaching. Eur J Esthet Dent. 2009;4(4):396403.

17. Borges AB, Torres CR, de Souza PA, Caneppele TM, Santos LF, Magalhaes AC . Bleaching gels containing calcium and fluoride: effect on enamel erosion susceptibility. Int J Dent. 2012;2012: 347848. doi:10.1155/2012/347848.

18. Cavalli V, Arrais CA, Giannini M, Ambrosano GM. Highconcentrated carbamide peroxide bleaching agents effects on enamel surface. J Oral Rehab. 2004;31(2):155-159.

19. Canay Ş, Çehreli MC. The effect of current bleaching agents on the color of light-polymerized composites in vitro. J Prosthet Dent. 2003;89(5):474-478.

20. Rosentritt M, Lang R, Plein T, Behr M, Handel G. Discoloration of restorative materials after bleaching application. Quintessence Int. 2005;36(1):33-39.

21. Ledoux WR, Malloy RB, Hurst RV V, McInnes-Ledoux P, Weinberg R. Structural effects of bleaching on tetracyclinestained vital rat teeth. J Prosth Dent. 1985;54(1):55-59. doi:10.1016/s0022-3913(85)80070-6.

22. Markovic L, Jordan RA, Lakota N, Gaengler P. Micromorphology of enamel surface after vital tooth bleaching. J Endod. 2007;33(5):607-610. doi:10.1016/j. joen.2007.01.011.

23. Summitt JB, Robbins JW, Hilton TJ, Schwartz RS. Fundamentals of operative dentistry: a contemporary approach. 3rd ed. USA: Quintessence; 2006.

24. Attin T, Muller T, Patyk A, Lennon AM. Influence of different bleaching systems on fracture toughness and hardness of enamel. Oper Dent. 2004;29(2):188-195.

25. Moritz AF, Beer F, Goharkhay K, Schoop U, Strassl M. Oral Laser Appl. Hanover Park, USA: Quintessence; 2006, . 